\title{
Effectiveness of Fuyuan Xingnao Decoction for patients with diabetes mellitus combined cerebral infarction: Erratum
}

In the article, "Effectiveness of Fuyuan Xingnao Decoction for patients with diabetes mellitus combined cerebral infarction", ${ }^{[1]}$ which appeared in Volume 98, Issue 39 of Medicine, the institute information of Zhen Ma is Xi'an Traditional Chinese Medicine Hospital. However, the correct information should be Xi'an Hospital of Traditional Chinese Medicine.

\section{Reference}

[1] Jiang C, Wang T, Ma Z. Effectiveness of Fuyuan Xingnao Decoction for patients with diabetes mellitus combined cerebral infarction. Medicine. 98;39: e17273. 